\title{
Vulnerabilidad de los sistemas de polinización de cactáceas columnares de México
}

\author{
Vulnerability of pollination systems of columnar cacti of Mexico
}

\author{
ALFONSO VALIENTE-BANUET
}

Instituto de Ecología, Universidad Nacional Autónoma de México, Apartado Postal 70-275, México 04510, Distrito Federal; e-mail: avali@servidor.unam.mx

\begin{abstract}
RESUMEN
Se presenta un análisis geográfico sobre el grado de vulnerabilidad de los sistemas de polinización de cactáceas columnares de México que muestran un síndrome de polinización quiropterófila. Se partió del supuesto que sistemas especializados de polinización serían más vulnerables a la perturbación humana que los generalistas. Los resultados indican que las especies que se ubican en el límite norte de la distribución de las cactáceas columnares muestran patrones generalistas de polinización que las hace menos vulnerables a las perturbaciones que las que habitan el centro de México que presentan sistemas de interacción especializados. Este patrón contrastante podría estar relacionado con los movimientos migratorios de los murciélagos en el norte de la distribución de las cactáceas que pudo haber restringido la respuesta hacia la especialización, en tanto que poblaciones residentes de murciélagos en el centro de México podrían haber favorecido la especialización local.
\end{abstract}

Palabras clave: cactáceas columnares, especialización, murciélagos nectarívoros, polinización, tribu Pachycereeae.

\begin{abstract}
An analysis of the degree of vulnerability of the pollination systems of Mexican columnar cacti showing a chiropterophyllous pollination syndrome was conducted, assuming that specialized pollination interactions would be more vulnerable to human perturbations. The results indicate that the species inhabiting the northern distribution limit of the columnar cacti show generalized pollination systems whereas species inhabiting central Mexico showing specialized pollination interactions would be more vulnerable to perturbations. This contrasting geographic pattern might be related to the migratory movements of the nectar-feeding bats in the northern limit of distribution of the columnar cacti that restricted local specialization, whereas resident nectar-feeding bat populations in south-central Mexico probably favored local specialization.
\end{abstract}

Key words: columnar cacti, nectar feeding bats, pollination, specialization, tribe Pachycereeae.

\section{INTRODUCCIÓN}

La polinización de plantas con flores por animales representa uno de los procesos más importantes de la biología reproductiva de cerca del $90 \%$ de las especies de angiospermas (Kearns et al. 1998). Recientemente se ha argumentado que los sistemas de polinización se encuentran en una crisis creciente que tiene como base la fragmentación de los hábitats, cambios en el uso del suelo, prácticas de agricultura moderna, uso de pesticidas y herbicidas e invasión de plantas y animales no nativos a los ecosistemas naturales (Kearns et al. 1998). La llamada "crisis de la polinización", dada por el decremento de las poblaciones de vectores animales por procesos de perturbación humana, afecta no sólo a procesos ecológicos y evolutivos sino también la producción y rendimiento de cultivos que requieren forzosamente de la presencia de vectores animales. De hecho se calcula que a nivel global los beneficios económicos que esta interacción provee a la humanidad son alrededor de 112 billones de dólares americanos anuales (Costanza et al. 1997). Sin embargo, y a pesar de la enorme importancia que tiene el proceso de la polinización principalmente por insectos en cultivos agrícolas, el uso de pesticidas se ha incrementado hasta el presente (Kearns et al. 1998).

En el presente trabajo se sostiene que la vulnerabilidad de los sistemas de polinización a perturbaciones humanas dependerá del grado de especialización que mantiene la interacción plantapolinizador. Así cualquier perturbación que afecte el comportamiento o diezme a las poblaciones de polinizadores en sistemas especializados sería más vulnerable, por lo que el conocimiento de historia natural de los diferentes sistemas de po- 
linización sería la base para poder buscar alternativas de gestión y restauración de estos sistemas.

Tradicionalmente la biología de la polinización ha estado influida por la hipótesis del polinizador más eficiente y la especialización (Stebbins, 1970). Esta idea central en los sistemas de polinización proviene del trabajo pionero de Darwin publicado en 1862, quien señaló que las especies podían desarrollar por selección natural, interacciones especializadas cuyas últimas consecuencias podrían derivar en procesos de evolución recíproca (Thompson 1994). El mismo Darwin visualizó que la especialización hacia algún polinizador radicaba en la eficiencia de los mismos lo cual a su vez podría redundar en la evolución de caracteres morfológicos y/o fisiológicos florales. Así por ejemplo, la especialización está implícita en el concepto de "síndromes de polinización" los cuales son considerados como un conjunto de rasgos florales que reflejan adaptaciones hacia algún tipo de polinizador (Waser et al. 1996).

En trabajos recientes este principio organizador en los estudios de la biología de la polinización bajo el cual las interacciones entre plantas y polinizadores animales tienden a ser especializadas ha sido reconsiderado, al encontrarse que los sistemas generalistas son tan comunes como los especializados (Herrera 1996, Roubik 1992, Waser et al. 1996, Gómez \& Zamora 1999).

Uno de los grupos de angiospermas que han sido definidos como altamente especializados en sus sistemas de polinización son las Cactaceae, cuyas flores están fuertemente asociadas con animales (Porsch 1939, Grant \& Grant 1979, Valiente-Banuet et al. 1996). Así, las relaciones planta-polinizador han sido citadas como especializadas para diferentes especies del género Opuntia y su relación con abejas (Grant \& Hurd 1979); para la interacción entre colibríes y especies de géneros como Rathbunia, Peniocereus, Nopalea y Pachycereus (Gibson \& Nobel 1986); esfíngidos y especies de los géneros Epiphyllum, Cereus, Trichocereus, Acanthocereus y Selenicereus (Grant \& Grant 1979) y entre murciélagos nectarívoros y cactáceas columnares de los géneros Pachycereus y Stenocereus pertenecientes a la tribu Pachycereeae (Valiente-Banuet et al. 1996).

En este trabajo se analizan en una perspectiva geográfica los sistemas de polinización de cactáceas columnares con síndrome de polinización quiropterófilo pertenecientes a la tribu Pachycereeae, con el objetivo de determinar su vulnerabilidad frente a perturbaciones de origen humano, partiendo del hecho de que sistemas de polinización generalistas serían menos vulnerables que los especializados.

\section{CACTÁCEAS COLUMNARES Y MURCIÉLAGOS NECTARÍVOROS}

La tribu Pachycereeae (sensu Gibson \& Horak 1978) está constituida por 70 especies distribuidas principalmente en México con algunas especies presentes en Venezuela (Gibson \& Nobel 1986, Valiente-Banuet et al. 1996). Aproximadamente un $70 \%$ de las especies presentan un síndrome de polinización quiropterófilo (sensu Faegri \& van der Pijl 1979), caracterizado por flores grandes de antésis nocturna, con periantos blanquecinos que producen grandes cantidades de polen y néctar y olores desagradables (ValienteBanuet et al. 1996, Valiente-Banuet \& GodínezÁlvarez en prensa). Varias de estas especies constituyen el componente dominante de vegetación árida y semiárida de México, siendo la región central del país, que incluye la cuenca del Río Balsas y el Valle de Tehuacán-Cuicatlán, la zona considerada como el principal centro de diversificación de la tribu con 45 de las 70 especies (Valiente-Banuet et al. 1996). En un sentido latitudinal la diversidad de las cactáceas columnares disminuye progresivamente partiendo del centro de México a lo largo de dos franjas, una que corre a lo largo de la vertiente del Océano Pacífico llegando al sudoeste de los Estados Unidos en donde hay seis especies registradas y otra a lo largo de la vertiente del Golfo de México con un total de cinco especies en el nordeste de México (Valiente-Banuet et al. 1996).

La distribución de las Pachycereeae muestra el mismo patrón geográfico que los murciélagos nectarívoros (Phyllostomidae: Glossophaginae), caracterizado por una alta concentración de nueve especies en la parte central de México desde el Eje Neovolcánico hacia la cuenca del Río Balsas y el Valle de Tehuacán-Cuicatlán (ValienteBanuet et al. 1996). La diversidad disminuye conforme se avanza latitudinalmente hacia el norte en dos franjas que corren por las vertientes del Océano Pacífico y el Golfo de México (ValienteBanuet et al. 1996). Tres de estas especies, Leptonycteris curasoae yerbabuenae, Leptonycteris nivalis y Choeronycteris mexica$n a$, son altamente dependientes del consumo de recursos florales y han sido consideradas como migratorias latitudinales (Koopman 1981, Cockrum 1991, Fleming et al. 1993).

\section{LOS POLINIZADORES DE LAS CACTÁCEAS COLUMNARES}

Son dos las áreas geográficas de México que han sido estudiadas detalladamente dentro del rango 
de distribución de las Pachycereeae. La primera, fuera de la línea del Trópico de Cáncer localizada en el Desierto Sonorense ( $\left.28^{\circ} 50^{\prime} \mathrm{N}\right)$, tres especies de cactáceas columnares Carnegiea gigantea, Pachycereus pringlei y Stenocereus thurberi producen flores quiropterófilas durante la primavera por periodos de hasta $18 \mathrm{~h}$ y son visitadas durante la noche por Leptonycteris curasoae y durante el día por aves y abejas (Fleming et al. 1996). En la segunda zona intertropical, el Valle de TehuacánCuicatlán $\left(18^{\circ} 20^{\prime} \mathrm{N}\right)$, las especies Neobuxbaumia tetetzo, N. mezcalaensis, N. macrocephala, Pachycereus weberi y Stenocereus stellatus producen flores nocturnas quiropterófilas durante la primavera y parte del verano y son visitadas por tres especies de murciélagos nectarívoros $L$. curasoae, L. nivalis y Choeronycteris mexicana. Las flores permanecen abiertas por periodos de 14 h y son visitadas principalmente por aves e insectos (Valiente-Banuet et al. 1996, 1997a, 1997b).
Por el contrario, el número de visitantes diurnos y nocturnos es significativamente superior en las cactáceas columnares del norte de México y en dos de las tres especies estudiadas la tasa de fructificación producida por polinizadores diurnos alcanza valores entre 58 y el $81 \%$ (Tabla 1 ). Al analizar las especies que habitan el centro de México el número de visitantes diurnos y nocturnos es mucho menor y dos de las tres especies consideradas dependen exclusivamente de la polinización por murciélagos, con tasas de fructificación superiores al $95 \%$, en tanto que la tercera especie presenta una tasa de fructificación del 90 $\%$ correspondiente a murciélagos y de un $10 \%$ por aves (Tabla 1). Esta información indica que existe un patrón geográfico contrastante en el cual las cactáceas columnares en el límite norte de su distribución (Desierto Sonorense) son polinizadas por un amplio grupo de vectores animales y entre los que sobresalen los organismos

TABLA 1

Resumen de los visitantes florales y polinizadores efectivos de las cactáceas columnares de México: tomado de Alcorn et al. (1959, 1961, 1962), Fleming et al. (1994, 1996), Valiente-

Banuet et al. (1996, 1997a, 1997b), Casas et al. (1999). Especies de visitantes y polinizadores: murciélagos: Leptonycteris curasoae (1), L. nivalis (2), Choeronycteris mexicana (3),

Artibeus jamaicensis (4), A. intermedius (5); Glossophaga soricina (6), Antrozous pallidus

(7); aves: Calypte costae (8), Zenaida macroura (9), Z. asiatica (10), Melanerpes uropygialis (11), M. hypopolius (12), Colaptes auratus (13), Toxostoma curvirostre (14), Auriparus flaviceps (15), Psaltriparus minimus (16), Carpodacus mexicanus (17), Amazilia violiceps (18), Calothorax lucifer (19), Cynanthus latirostris (20), C. sordidus (21); insectos nocturnos: Hyles lineata (22), Eumorpha anchemola (23); insectos diurnos: Apis mellifera (24), Melipona sp. (25), Trigona sp. (26), Carpophylus sp. (27), Bombus sp. (28), Melipona sp. (29)

Flower visitors and effective pollinators of the columnar cacti of Mexico. Sources: Alcorn et al. (1959, 1961, 1962), Fleming et al. (1994), (1996), Valiente-Banuet et al. (1996), (1997a), (1997b), Casas et al. (1999). Visitors and pollinators: bats: Leptonycteris curasoae (1), L. nivalis (2), Choeronycteris mexicana (3), Artibeus jamaicensis (4), A. intermedius (5); Glossophaga soricina (6), Antrozous pallidus (7); birds: Calypte costae (8), Zenaida macroura (9), Z. asiatica (10), Melanerpes uropygialis (11), M. hypopolius (12), Colaptes auratus (13), Toxostoma curvirostre (14), Auriparus flaviceps (15), Psaltriparus minimus (16), Carpodacus mexicanus (17), Amazilia violiceps (18),

Calothorax lucifer (19), Cynanthus latirostris (20), C. sordidus (21); nocturnal Insects: Hyles lineata (22), Eumorpha anchemola (23); diurnal insects: Apis mellifera (24), Melipona sp. (25), Trigona sp. (26), Carpophylus sp. (27), Bombus sp. (28), Melipona sp. (29)

\begin{tabular}{|c|c|c|c|}
\hline Especie & Latitud & Visitantes florales & Polinizadores efectivos (fruit set) \\
\hline Carnegiea gigantea & $28^{\circ} 50^{\prime} \mathrm{N}$ & $\begin{array}{c}1,7,8,9,10,11,13,14,15 \\
16,17,22,24\end{array}$ & $\begin{array}{c}\text { Abejas }(58 \%) \text {, murciélagos }(54 \%) \\
\text { y aves }(34 \%)\end{array}$ \\
\hline Pachycereus pringlei & $28^{\circ} 50^{\prime} \mathrm{N}$ & $\begin{array}{c}1,7,8,9,10,11,13,14,15 \\
16,17,22,24\end{array}$ & $\begin{array}{c}\text { Murciélagos (89 \%), aves (66 \%) } \\
\text { y abejas }(14 \%)\end{array}$ \\
\hline Stenocereus thurberi & $28^{\circ} 50^{\prime} \mathrm{N}$ & $\begin{array}{c}1,7,8,9,10,11,13,14,15 \\
16,17,22,24\end{array}$ & $\begin{array}{c}\text { Aves }(81 \%) \text {, murciélagos }(38 \%) \\
\text { y abejas }(19 \%)\end{array}$ \\
\hline Neobuxbaumia mezcalaensis & $18^{\circ} 20^{\prime} \mathrm{N}$ & $1,2,3,21,23,24,26$ & Murciélagos $(90 \%)$ y aves $(10 \%)$ \\
\hline Neobuxbaumia tetetzo & $18^{\circ} 20^{\prime} \mathrm{N}$ & $1,3,4,12,18,21,24$ & Murciélagos (98\%) \\
\hline Neobuxbaumia macrocephala & $18^{\circ} 20^{\prime} \mathrm{N}$ & $1,2,3,21,23,24,26$ & Murciélagos (95\%) \\
\hline Pachycereus weberi & $18^{\circ} 20^{\prime} \mathrm{N}$ & $1,2,3,4,5,20,24,26$ & Murciélagos \\
\hline Stenocereus stellatus & $18^{\circ} 20^{\prime} \mathrm{N}$ & $\begin{array}{c}1,2,3,4,12,18,20,21,24 \\
25,26,27,28,29\end{array}$ & MurciélagoS \\
\hline
\end{tabular}


de actividad diurna, en tanto que las cactáceas del centro de México son polinizadas exclusivamente por murciélagos nectarívoros. La dependencia exclusiva de murciélagos para la polinización de cactáceas columnares que muestran un síndrome de polinización quiropterófilo ha sido reportada también para especies que habitan Sudamérica en Venezuela (Nassar et al. 1997).

\section{SISTEMAS GENERALISTAS VERSUS ESPECIALISTAS}

El sistema de polinización generalista en el límite norte de la distribución de las Pachycereeae podría estar relacionado con la impredecibilidad del recurso polinizador en un contexto geográfico, que restringe la respuesta hacia la especialización a polinizadores particulares. El murciélago Leptonycteris curasoae yerbabuenae, considerado como el principal polinizador de las cactáceas columnares (Valiente-Banuet et al. 1996, 1997a, 1997b, Casas et al. 1999) es una especie cuya biología ha sido analizada de acuerdo a un supuesto patrón de migración latitudinal a todo lo largo de su distribución, en el que supuestamente todas las poblaciones de la especie se mueven desde el sudoeste de los Estados Unidos hacia el sur durante el otoño e invierno regresando al mismo sitio durante la primavera y el verano. (Cockrum 1991, Fleming et al. 1993, Wilkinson \& Fleming 1996). Sin embargo, estudios recientes indican que no existe un patrón generalizado de la migración y que solamente las poblaciones ubicadas alrededor de la latitud $29^{\circ} \mathrm{N}$ se desplazan estacionalmente hacia el sur (Cockrum 1991, Rojas-Martínez et al. 1999), lo cual lleva consigo una alta fluctuación en sus densidades poblacionales. Estos movimientos de las poblaciones norteñas podrían estar relacionados con la disponibilidad de recursos alimenticios y las bajas temperaturas durante el otoño y el invierno (Rojas-Martínez et al. 1999). En tanto, L. curasoae tiene poblaciones residentes en la cuenca del Río Balsas y el Valle de Tehuacán-Cuicatlán, en el centro de México (Rojas-Martínez et al. 1999) en donde existen recursos alimenticios durante todo el año a diferentes altitudes, por lo que movimientos altitudinales entre los 900 y los $1.700 \mathrm{~m}$, en vez de latitudinales, serían suficientes para que estos animales aseguraran su permanencia en esta área del país, donde los murciélagos mantienen una estrecha interacción con las cactáceas columnares (Valiente-Banuet et al. 2000). Estas diferencias geográficas en la disponibilidad de polinizadores a través del rango de distribución de las cactáceas columnares podría explicar por qué los cactus extratropicales tienen una mayor dependencia de aves e insectos (Fleming et al. 1996) que de murciélagos, definiendo un sistema generalista de polinización y uno especializado en el centro de México. Diversos trabajos han señalado que una alta variabilidad en la distribución y abundancia de las especies polinizadoras puede restringir la respuesta hacia la especialización a polinizadores particulares (Horvitz \& Schemske 1990, Gómez \& Zamora 1999), y por el contrario una estructura geográfica de especialización puede ocurrir cuando la predecibilidad en la interacción es alta, resultando en especialización local (Waser et al. 1996). Estas observaciones indican que las cactáceas columnares que habitan el centro de México son más vulnerables a la "crisis de la polinización" señalada anteriormente, por lo que la perturbación de las poblacionales de los vectores de polen en los trópicos tendría un mayor efecto sobre la adecuación de las cactáceas. Esto enfatiza la importancia de la protección de los mutualistas a estas latitudes que permita que los procesos de reproducción de las plantas no se vean afectadas por la perturbación.

\section{LOS RETOS PARA LA CONSERVACIÓN}

La llamada "crisis de la polinización" tiene como causas la desaparición o disminución de las densidades poblacionales de vectores de polen en diversos grupos de angiospermas (Kearns et al. 1998). En el caso de los sistemas cactus columnarpolinizador en el centro de México son dos los principales riesgos que afectan las densidades de polinizadores: la destrucción de grandes áreas con vegetación natural dominadas por cactáceas columnares con fines agrícolas y la perturbación de refugios de murciélagos nectarívoros. En cualquier caso, el estrategia de conservación deberá basarse en un enfoque a nivel del ecosistema que permita la conservación de grandes grupos de mutualistas, más que la conservación de ciertas especies en particular. Las cactáceas columnares son sin duda una fuente de alimento muy importante para murciélagos nectarívoros y la disminución de las densidades de éstas tienen importantes repercusiones en los sistemas de polinización. Así por ejemplo, grandes densidades de murciélagos nectarívoros dependen de la vegetación dominada por cactáceas columnares en la isla de Cusaçao (Petit \& Pors 1996) y un total de 33 especies de plantas pertenecientes a las familias Agavaceae, Bignoniaceae Convolvulaceae y Cactaceae proveen de alimento a los murciélagos durante prácticamente todo el año en el centro de México (Rojas-Martínez et al. 1999). Estas espe- 
cies de plantas constituyen el componente dominante de por lo menos 9 asociaciones vegetales en el Valle de Tehuacán-Cuicatlán (Valiente-Banuet et al. 2000), cuya conservación debe ser una prioridad.

La destrucción de refugios utilizados por los murciélagos nectarívoros por los pobladores locales tiene como base el hecho de que éstos los confunden con el vampiro Desmodus rotundus, especie que afecta fuertemente al ganado. Este vampiro transmite enfermedades como tripanosomiasis bovina y equina y el virus de la rabia paralítica, enfermedades a las que se les asocia pérdidas de hasta un millón de cabezas al año en Latinoamérica (Thompson et al. 1972). La asesoría a pobladores locales acerca de la diversidad de murciélagos debe ser crucial para disminuir el riesgo de los refugios de los murciélagos nectarívoros. Prácticas de difusión ya realizadas en lugares específicos cercanos a refugios importantes en el centro de México, han permitido disminuir las prácticas destructivas realizadas cotidianamente por los habitantes, los cuales ahora son capaces de reconocer refugios habitados por vampiros distinguiéndolos de los de otras especies. En todo caso, el problema principal aún persiste y en la medida en que no se cuente con un método de control de las poblaciones de vampiro, no será posible lograr una protección adecuada de los sistemas de polinización más vulnerables como lo son los intertropicales.

\section{AGRADECIMIENTOS}

Agradezco la invitación y apoyos otorgados por parte de Anna Traveset y Mary Kalin Arroyo para participar en el taller "Aspectos Teóricos de la Reproducción en Plantas: Consecuencias para la Conservación", bajo el programa Iberoamericano de Ciencia y Tecnología para el Desarrollo (CYTED). Alfonso Torres me proporcionó información sobre Desmodus rotundus en México. Los estudios sobre biología reproductiva en cactáceas se han hecho bajo el apoyo del programa PAPIIT (IN-207798), el Fondo Mexicano para la Conservación de la Naturaleza y la Comisión Nacional para el Conocimiento y Uso de la Biodiversidad.

\section{LITERATURA CITADA}

ALCORN SM, SE MCGREGOR, GD BUTLER \& EB KURTZ (1959) Pollination requirements of the saguaro (Carnegiea gigantea). Cactus and Succulent Journal 31: 39-41.
ALCORN SM, SE MCGREGOR \& G OLIN (1961) Pollination of Saguaro cactus by doves, nectar-feeding bats and honey bees. Science 132: 1594-1595.

ALCORN SM, SE MCGREGOR \& G OLIN (1962) Pollination requirements of the Organpipe cactus. Cactus and Succulent Journal 34: 134-138.

CASAS A, A VALIENTE-BANUET, A ROJASMARTÍNEZ \& P DÁVILA (1999) Reproductive biology and the process of domestication of the columnar cactus Stenocereus stellatus in central Mexico. American Journal of Botany 86: 534-542.

COCKRUM EL (1991) Seasonal distribution of northwestern populations of the long-nosed bat Leptonycteris sanbornii family Phyllostomidae. Anales del Instituto de Biología, Universidad Nacional Autónoma de México, Serie Zoología 62: 181-202.

COSTANZA R, R D'ARGE, R DE GROOT, S FARBER \& M GRASSO (1997) The value of the world's ecosystem services and natural capital. Nature 387: 253-260.

FAEGRI K \& L VAN DER PIJL (1979) The principles of pollination ecology. Tercera edición. Pergamon Press, Oxford, Inglaterra. $244 \mathrm{pp}$.

FLEMING TH, RA NUÑEZ \& LSL STEMBERG (1993) Seasonal changes in the diets of migrant and nonmigrant nectarivorous bats as revealed by carbon stable isotope analysis. Oecologia 94: 72-75.

FLEMING TH, MD TUTTLE \& MA HORNER (1996) Pollination biology and the relative importance of nocturnal and diurnal pollinators in three species of sonoran desert columnar cacti. Southwestern Naturalist 41: 257-269.

GIBSON AC \& KE HORAK (1978) Systematic anatomy and phylogeny of Mexican columnar cacti. Annals of the Missouri Botanical Garden 65: 999-1057.

GIBSON AC \& PS NOBEL (1986) The cactus primer. Harvard University Press, Cambridge, Massachusetts. $286 \mathrm{pp}$.

GÓMEZ JM \& R ZAMORA (1999) Generalization vs. specialization in the pollination system of Hormatophylla spinosa (Cruciferae). Ecology 80: 796805.

GRANT V \& KA GRANT (1979) The pollination spectrum in the southwestern american cactus flora. Plant Systematics and Evolution 133: 29-37.

GRANT V \& PD HURD (1979) Pollination of the southwestern Opuntias. Plant Systematics and Evolution 133: 15-28.

HERRERA CM (1996) Floral traits and plant adaptations to insect pollinators: a devil's advocate approach. En: Barret SCH \& DG Lloyd (eds) Floral biology: 65-87. Chapman \& Hall, New York, New York.

HOR VITZ CC \& DW SCHEMSKE (1990) Spatiotemporal variation in insect mutualisms of a neotropical herb. Ecology 71: 1085-1097.

KEARNS CA, DW INOUYE \& NM WASER (1998) Endangered mutualisms: the conservation of plantpollinator interactions. Annual Review of Ecology and Systematics 29: 83-112.

KOOPMAN KF (1981) The distributional patterns of new world nectar-feeding bats. Annals of the Missouri Botanical Garden 68: 352-369. 
NASSAR JM, N RAMÍREZ \& O LINARES (1997) Comparative pollination biology of Venezuelan columnar cacti and the role of nectar-feeding bats in their sexual reproduction. American Journal of Botany 84: 918-927.

PETIT S \& L PORS (1996) Survey of columnar cacti and carrying capacity for nectar-feeding bats in Curacao. Conservation Biology 10: 769-775.

PORSCH O (1939) Das Bestäubungsleben der Kakteenblüte. II. Cactaceae. Jahrbuch der Deutsche Kakteen-Gesellschaft 2: 81-142.

ROJAS-MARTÍNEZ A, A VALIENTE-BANUET, A ARIZMENDI, A ALCÁNTARA-EGÚREN \& H ARITA (1999) Seasonal distribution of the longnosed bat (Leptonycteris curasoae) in North America: does a generalized migration pattern really exist? Journal of Biogeography 26: 1065-1077.

ROUBIK DW (1992) Loose niches in tropical communities: why are there so few bees and so many trees? En: Hunter MD, Ohgushi T \& PW Price (eds) Effects of resource distribution on animal-plant interactions. Academic Press, San Diego, California.

STEBBINS GL (1970) Adaptive radiation of reproductive characteristics in Angiosperms, I: pollination mechanisms. Annual Reviews of Ecology and Systematics 1: 307-326.

THOMPSON JN (1994) The coevolutionary process. University of Chicago Press, Chicago, Illinois. 376 pp.

THOMPSON RD, GC MITCHELL \& RJ BURNS (1972) Vampirebat control systemic treatment of livestock with an anticoagulant. Science 177: 806.
VALIENTE-BANUET A, MC ARIZMENDI, A ROJASMARTÍNEZ \& L DOMÍNGUEZ-CANSECO (1996) Ecological relationships between columnar cacti and nectar feeding bats in Mexico. Journal of Tropical Ecology 12: 103-119.

VALIENTE-BANUET A, A ROJAS-MARTÍNEZ, MC ARIZMENDI \& P DÁVILA (1997a) Pollination biology of two columnar cacti (Neobuxbaumia mezcalaensis and Neobuxbaumia macrocephala) in the Tehuacán Valley, central Mexico. American Journal of Botany 84: 452-455.

VALIENTE-BANUET A, A ROJAS-MARTÍNEZ, A CASAS, MC ARIZMENDI \& P DÁVILA (1997b) Pollination biology of two winter-blooming giant columnar cacti in the Tehuacán Valley, central Mexico. Journal of Arid Environments 37: 331-341.

VALIENTE-BANUET A \& H GODÍNEZ-ÁLVAREZ (in press) Population and community ecology. En: Nobel PS (ed) Cacti: biology and uses. University of California Press.

VALIENTE-BANUET A, A CASAS A, P DÁVILA, MC ARIZMENDI, JL VILLASEÑOR \& J ORTEGARAMÍREZ (2000) La vegetación del Valle de Tehuacán-Cuicatlán. Boletín de la Sociedad Botánica de México 67: 24-74.

WASER NM, L CHITTKA, MV PRICE, NM WILLIAMS \& J OLLERTON (1996) Generalization in pollination systems, and why it matters. Ecology 77: 1043-1060.

WILKINSON GS \& TH FLEMING (1996) Migration and evolution of lesser long-nosed bat Leptonycteris curasoae, inferred from mitocondrial DNA. Molecular Ecology 5: 329-339. 